\title{
Implicações do ambiente, condições e organização do trabalho na saúde do professor: uma revisão sistemática
}

\author{
Implications of the environment, conditions and organization \\ of work on teacher health: a systematic review
}

Jaqueline Galleazzi da Luz (https://orcid.org/0000-0003-4639-6752) ${ }^{1}$

Sergio Luiz Ribas Pessa (https://orcid.org/0000-0001-9765-6572) ${ }^{1}$

Roger Poglia da Luz (https://orcid.org/0000-0003-4739-0467) ${ }^{1}$

Fernando José Avancini Schenatto (https://orcid.org/0000-0002-3717-2370) ${ }^{1}$

${ }^{1}$ Universidade Tecnológica Federal do Paraná. R. Via do Conhecimento km 01, Fraron. 85503-390 Pato Branco PR Brasil. jgalleazzi@utfpr.edu.br

\begin{abstract}
The state of health of the teacher is fundamental to the success of the teaching process. In addition to the intrinsic aspects, the scholastic context affects the well-being of the educator. The objective of this study was to identify the main psychosocial, structural and relational factors in the teaching profession. A systematic review of the literature, with the use of electronic research in eight databases, identified 2,479 articles on the topic published between 1997 and 2016. Metric and subjective parameters were adopted for selection in this portfolio and, in all, 32 publications were eligible for analysis, of which 29 were published in English. After the bibliometric and content characterization of the final portfolio, the main results of strain were psychosomatic disorders, with an emphasis on stress and the Burnout Syndrome, as well as voice disturbances. Workload, interpersonal relations and conditions of the scholastic environment are highlighted as the major agents of these conditions. Changes and adaptations in classrooms, actions of prevention and social support are pointed out as possible solutions to guarantee the quality of life of these professionals.

Key words Teachers, Occupational health, Work environment, Working conditions
\end{abstract}

Resumo O estado da saúde do professor é fundamental para o êxito do processo de ensino. Além dos aspectos intrínsecos, o contexto escolar afeta o bem-estar do educador. O objetivo deste estudo foi identificar os principais fatores psicossociais, estruturais e relacionais na profissão docente. Uma revisão sistemática da literatura, com o uso de pesquisa eletrônica em oito bases de dados, identificou 2.479 artigos sobre o tema, publicados entre 1997 e 2016. Adotaram-se parâmetros métricos e subjetivos para a seleção nesse portfolio e, ao todo, 32 publicações restaram elegíveis para análise, das quais 29 foram publicadas na lingua inglesa. Transcorrida a caracterização bibliométrica e de conteúdo do portfolio final, os trabalhos apontaram, como principais resultados de desgaste, os transtornos psicossomáticos, com ênfase para o estresse e a Síndrome de Burnout, além dos distúrbios da voz. A carga de trabalho, as relações interpessoais e as condições do ambiente escolar são destacadas como os maiores agentes dessas enfermidades. Mudanças e adequações de salas de aula, ações de prevenção e suporte social são apontadas como possíveis soluções para garantir a qualidade de vida desses profissionais.

Palavras-chave Docentes, Saúde do trabalhador, Ambiente de trabalho, Condições de trabalho. 


\section{Introdução}

O ambiente educacional pode ser considerado como múltiplo, complexo, dinâmico e interativo $^{1,2}$. Para se encaixar neste contexto multifacetado, o professor, elemento fundamental no processo educativo, deve estar sempre acessível às mudanças.

Neste cenário, torna-se desafiador o desempenho da atividade docente. Lidar com a inflexibilidade de horários, indisciplina em sala de aula, precariedade das condições existentes, burocracia administrativa, défice de recursos, situação salarial, entre outras adversidades, acarreta em sobrecarga de trabalho ${ }^{3-5}$. Essa volubilidade influencia nas atividades diárias do educador, uma vez que a intensificação de atribuições e responsabilidades, somadas às elevadas exigências do trabalho, implicam no aumento da carga de trabalho, e consequentemente, afetam o desempenho profissional dos professores ${ }^{6,7}$.

A satisfação laboral, por sua vez, está relacionada com o contentamento pessoal, características institucionais, motivações individuais e organizacionais ${ }^{8,9}$. Ainda, como complemento, tem-se a concepção de bem-estar no trabalho, termo associado com disposições individuais, ambiente, condições e excesso de trabalho, passível de suscitar o empenho e o desempenho docente ${ }^{10-14}$.

Os aspectos psicossociais, estruturais e relacionais intervêm no convívio e rotina de trabalho e, além destes, a organização e a vulnerabilidade das demandas de expediente resultam na desestabilização da capacidade para o trabalho ${ }^{15-17}$. Vários dos fatores citados podem acometer a saúde do professor e, por consequência, acarretar em diminuição da aptidão laboral.

Muitos estudos tratam, de maneira singular, a respeito dos efeitos do trabalho na saúde mental dos educadores, dentre os quais menciona-se a desvalorização profissional, baixa autoestima e ausência de resultados ${ }^{18}$. Possíveis origens de estresse também são relatadas, destacando-se limitações de tempo e recursos, relação com colegas de profissão, falta de reconhecimento, condições de trabalho e clima organizacional ${ }^{19,20}$. Uma doença mais grave, a Síndrome de Burnout, possui destaque nos acometimentos em docentes. Dentre as suas possíveis razões encontram-se o ambiente, a carga e a pressão no trabalho, carência de infraestrutura e falta de apoio, social e administrativo ${ }^{21-23}$.

A fala, instrumento essencial para o professor também está sujeita a lesões. Algumas das causas de alteração vocal são os ambientes ruidosos, uso extensivo da voz, falta de repouso e hidratação, qualidade do ar, fonação incorreta e alergias. Dentre os principais sintomas relatados estão a fadiga vocal, a rouquidão e a disfonia ${ }^{24-27}$.

Ainda como adversidade recorrente em docentes, tem-se a dor musculoesquelética. Entre as regiões mais afetadas estão as costas, os ombros, o pescoço e as extremidades ${ }^{28}$. Alguns dos fatores capazes de afetar a estrutura física são as posturas incorretas, os longos períodos na mesma posição, o mobiliário inadequado, além da falta de fortalecimento e alongamento ${ }^{16,22}$.

Como possíveis consequências do agravo da saúde do professor estão a aposentadoria precoce e o abandono da profissão $0^{30,31}$. Diante do exposto, torna-se necessário a investigação das circunstâncias causais e a procedente intervenção, em situações cabíveis de reparação, no ambiente, organização e condições do trabalho dos educadores, acarretando, por consequência, na melhoria da capacidade para o trabalho ${ }^{13,16}$. Logo, a presente revisão sistemática da literatura objetiva contribuir com a reunião de trabalhos científicos alusivos ao tema, identificação de métodos e instrumentos utilizados para aferir a saúde e as condições de trabalho docente, e constatação de quais são os principais fatores relacionados à saúde que acometem os educadores.

\section{Métodos}

Este estudo, de natureza aplicada e finalidade exploratória, utilizou a técnica de revisão sistemática da literatura, que busca reunir evidência empírica que se ajusta em critérios de elegibilidade pré-determinados para responder a uma pergunta específica de pesquisa, seus procedimentos são explícitos e reprodutíveis para identificar, selecionar, e avaliar trabalhos relevantes ${ }^{32}$.

\section{Estratégia de busca e fonte bibliográfica}

$\mathrm{O}$ trabalho foi elaborado em atendimento às recomendações da declaração PRISMA (Preferred Reporting Items for Systematic Reviews and Meta-Analyses), que consiste em uma lista de verificação de 27 itens e um diagrama de fluxo de quatro fases com o objetivo de auxiliar autores a melhorar a comunicação de revisões ${ }^{33}$.

Foi conduzida, nos meses de junho e julho de 2016, uma pesquisa eletrônica nas seguintes bases de dados: Biblioteca Virtual em Saúde (BVS), Cochrane, ERIC, United States National Library of Medicine (PubMED), SciELO, ScienceDirect, 
Scopus e Web of Science. Foram aplicados, nos campos title e/ou abstract + keywords, descritores de três constructos: saúde e carga de trabalho; ambiente de trabalho; e docência, o que resultou na chave de busca ((Ergonomics OR Health OR "Human factors" OR workload) AND ("Educational environment" OR "Job Conditions" OR "Organizational climate" OR "Work environment" OR "working conditions" OR "Working environment" OR workplace) AND ("Academic profession" OR "Academic staff” OR Professor OR Teacher OR “Teaching staff")).

\section{Critérios de seleção e identificação dos estudos}

Como critérios de inclusão, delimitaram-se o ano de publicação, a partir de 1997 e o formato da publicação, artigos em periódicos e revisões.

A primeira consulta retornou 2.479 artigos sendo: 1466 da Web of Science; 843 da Scopus; 109 da PubMed; 52 da Science Direct; 8 da Eric e 1 da Cochrane. As bases BVS e Scielo não retornaram artigos.

Como primeiro procedimento de exclusão, foram descartadas as publicações que estavam duplicadas, fruto da indexação do periódico em mais de uma base de dados e, após a aplicação desse filtro, restaram 1.928 trabalhos.

$\mathrm{Na}$ sequência, realizou-se a primeira triagem subjetiva, caracterizada pela adequação do título com o objetivo da pesquisa. Como resultado desta etapa, foram selecionados 265 trabalhos, que tratavam de estudos em ambientes de ensino ou com professores.

A próxima etapa da análise foi estabelecida com o subsídio de indicadores, identificando-se as métricas dos artigos e os respectivos periódicos. Foram consultados o Journal Citation Reports ${ }^{\circledR}$ (JCR), o SCImago Journal Rank (SJR), e o Google Acadêmico (http://scholar.google.com. br/). Para os periódicos, 140 constam no Google Acadêmico, 87 no JCR, e 136 no SJR. Com relação aos artigos, 213 dos 265 apresentaram pelo menos uma citação no Google Acadêmico.

Como critério de inclusão, utilizou-se a interseção dos dados resultantes nesses índices, ou seja, apenas os artigos/periódicos com registro no JCR, no SJR e no Google Acadêmico foram incluídos. Desta forma, restaram 133 artigos, incluindo 05 que não possuíam citações, mas foram selecionados por terem sido publicados em 2015 ou 2016.

Como segunda seleção subjetiva, realizou-se a leitura dos resumos dos artigos. Descartaramse trabalhos que tratavam sobre absenteísmo, assédio, estudos envolvendo, além de professores, alunos ou outras profissões como: mineiros, aeromoças, atendente de creche, cuidadores de crianças com necessidades especiais e idosos. Permaneceram assim, 92 artigos.

Para refinar a catalogação dos artigos, novamente recorreu-se às métricas dos índices. Calcularam-se as médias do JCR, SJR, Índice h5 e quantidade de citações. Passaram para a próxima etapa apenas os artigos que possuíam pelo menos 02 fatores acima da média. Mantiveram-se 35 artigos, dos quais: 12 possuem indicadores acima da média nos 4 índices; 9 possuem indicadores acima da média em 3 índices; e 14 possuem indicadores acima da média em 2 índices.

Procedeu-se então à leitura, na íntegra, dos 35 artigos; destes, 32 estavam alinhados com o objeto da pesquisa e compuseram o portfólio bibliográfico estudado. A Figura 1 demonstra o fluxo de seleção dos estudos.

\section{Extração dos dados}

A triagem dos artigos, assim como a análise dos dados, foi realizada de maneira independente, por dois revisores. Utilizou-se um formulário padronizado para extrair as informações relevantes para este trabalho. Analisaram-se os periódicos (métricas e procedência), os autores (instituição e país de origem), os artigos (ano de publicação; objetivo(s); país e local de realização do estudo; população e seleção amostral; desenho do estudo; instrumentos de coleta de dados; principais resultados e limitações identificadas).

\section{Resultados}

A elaboração deste trabalho, desde a definição de palavras-chave, busca em bases de dados, adoção de filtros, aplicação de critérios de inclusão e exclusão e leitura de títulos, resumos e textos completos, resultou na seleção dos 32 artigos $^{2-9,12,13,15,16,18-21,27,30,31,34-46}$ para análise.

\section{Caracterização bibliométrica do portfólio}

O período de publicações variou de 2002 a 2016, sendo que 07 trabalhos foram publicados a partir de 2010; 18 trabalhos foram publicados no intervalo de 2006 a 2009; e 07 trabalhos foram publicados entre 2002 e 2005, conforme apresentado na Tabela 1. Chama-se a atenção para o fato de que nenhuma publicação está compreendida entre 1997 e 2001. 


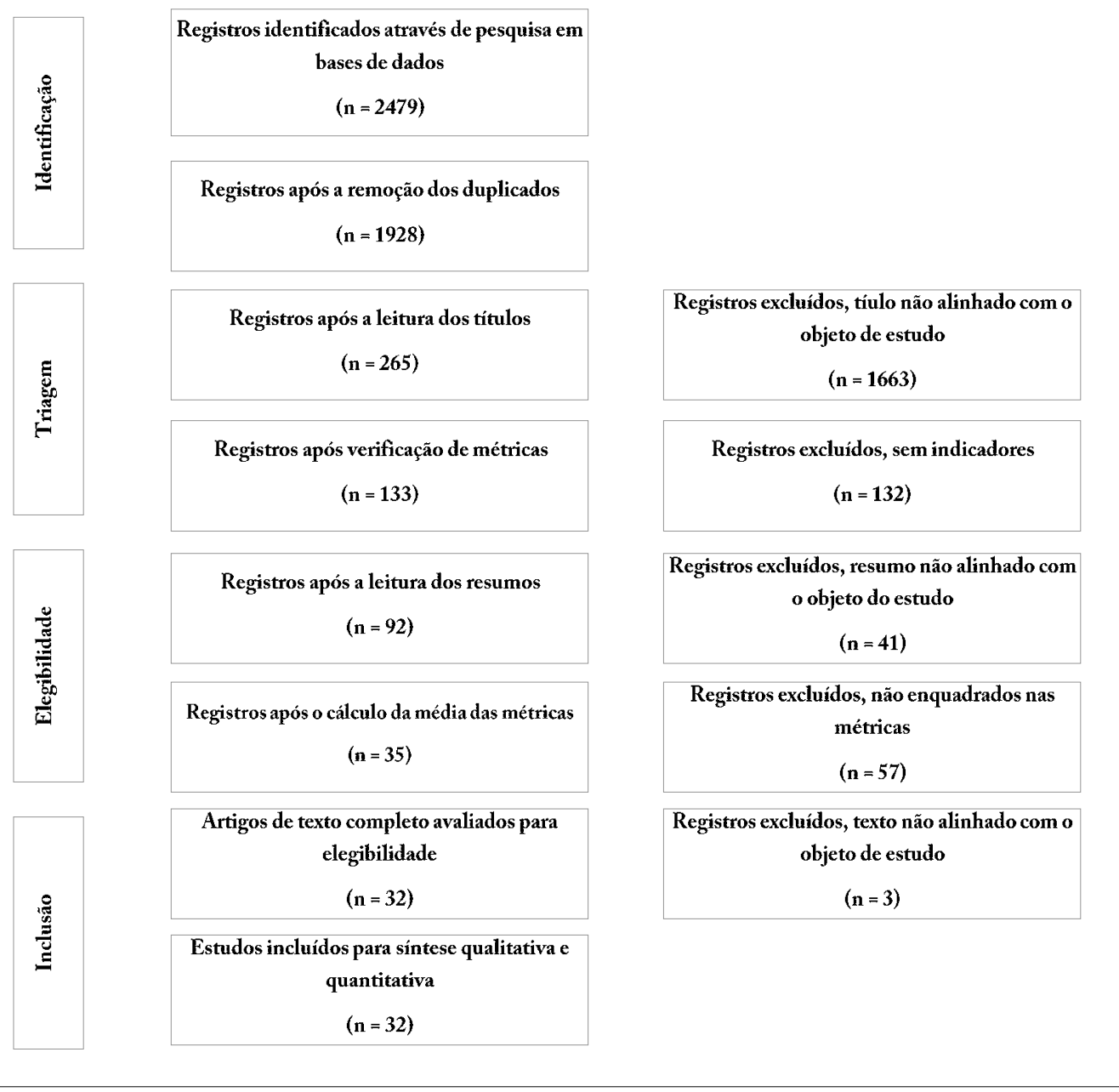

Figura 1. Fluxograma de seleção dos artigos incluídos no estudo.

Fonte: adaptado de Liberati et al..$^{32}$.

Os periódicos com maior número de artigos selecionados, e que concentram $50 \%$ das publicações, são: Journal of Voice, com 04 publicações; Cadernos de Saúde Pública, International Journal of Occupational and Environmental Health, Psychology \& Health e Teaching and Teacher Education, com 03 publicações cada. Os demais trabalhos são de 16 periódicos diferentes. As 21 revistas representadas são de apenas 05 países: Inglaterra, com 11; Estados Unidos, com 05; Holanda, com 03; Brasil e Finlândia com apenas 01 periódico cada.

Com relação às citações, 10 dos 32 artigos foram citados cem ou mais vezes, com destaque para os trabalhos de Delcor et al. ${ }^{18}$, com 301 citações, Jepson e Forrest ${ }^{30}$, com 210 citações e
Unterbrink et al..$^{31}$, com 185 citações. Outros 10 artigos possuem entre 56 e 92 citações; os demais possuem de 2 a 48 citações.

Os trabalhos reunidos, na sua totalidade, são do formato "artigo em periódico", dos quais 29 foram publicados na língua inglesa e 03 na língua portuguesa.

A autoria dos artigos remete a 139 autores, sendo 114 distintos. Com relação à colaboração, 04 autores colaboram com 03 artigos, sendo eles Joachim Bauer, Michael Wirsching, Ruth Pfeifer e Thomas Unterbrink; 17 autores em 02 artigos e 93 autores contribuem em apenas 01 artigo. Alusivo à quantidade de autores por artigo, 04 trabalhos possuem 08 ou mais autores; 13 trabalhos foram escritos por 04,05 ou 06 autores; e 15 tra- 
Tabela 1. Características bibliográficas dos trabalhos incluídos na revisão de literatura.

\begin{tabular}{|c|c|c|}
\hline Características & $\mathbf{n}$ & $\%$ \\
\hline \multicolumn{3}{|l|}{ Período de publicação } \\
\hline $2002-2005$ & 7 & 21,9 \\
\hline 2006-2009 & 18 & 56,3 \\
\hline $2010-2013$ & 4 & 12,5 \\
\hline 2014-2016 & 3 & 9,4 \\
\hline \multicolumn{3}{|l|}{ Autores por publicação } \\
\hline$\leq 3$ & 15 & 46,8 \\
\hline $4-6$ & 13 & 40,6 \\
\hline$\geq 7$ & 4 & 12,5 \\
\hline \multicolumn{3}{|l|}{ Autores por país } \\
\hline Brasil & 17 & 14,9 \\
\hline Alemanha & 16 & 14,0 \\
\hline Inglaterra & 11 & 9,6 \\
\hline Austrália & 10 & 8,8 \\
\hline China & 10 & 8,8 \\
\hline Estados Unidos & 9 & 7,9 \\
\hline Demais países & 41 & 36,0 \\
\hline \multicolumn{3}{|l|}{ Periódico de publicação } \\
\hline Journal of Voice & 4 & 12,5 \\
\hline Cadernos De Saúde Publica & 3 & 9,4 \\
\hline $\begin{array}{l}\text { International Journal of } \\
\text { Occupational and Environmental } \\
\text { Health }\end{array}$ & 3 & 9,4 \\
\hline Psychology \& Health & 3 & 9,4 \\
\hline Teaching and Teacher Education & 3 & 9,4 \\
\hline $\begin{array}{l}\text { Demais periódicos com } 1 \text { artigo } \\
\text { cada }\end{array}$ & 16 & 50 \\
\hline Total & 32 & 100,0 \\
\hline \multicolumn{3}{|l|}{ Periódicos por país } \\
\hline Inglaterra & 11 & 52,4 \\
\hline Estados Unidos & 5 & 23,8 \\
\hline Holanda & 3 & 14,3 \\
\hline Brasil & 1 & 4,8 \\
\hline Finlândia & 1 & 4,8 \\
\hline Total & 21 & 100,0 \\
\hline
\end{tabular}

Fonte: do Autor.

balhos foram escritos por 03 ou menos autores. Os 114 pesquisadores estão vinculados a instituições de 16 diferentes países. Com destaque para o Brasil, com 17 autores; Alemanha com 16 autores e Inglaterra com 11 autores.

Quanto às palavras-chave, 01 artigo apresentou 09 descritores; 10 artigos apresentaram 06 ou 07 termos; 17 artigos apresentaram de 03 a 05 ; e 04 artigos não apresentaram descritores na sua composição.
Na elaboração dos 27 artigos que utilizaram palavras-chave, foram usados 100 descritores distintos, com destaque para burnout, com 07 aparições; teachers, com 06; teacher e working conditions com 04 ocorrências cada.

\section{Caracterização de conteúdo do portfólio}

Quanto ao país de realização dos estudos, como pode ser observado na Tabela 2, destacamse o Brasil, com 05 estudos, Alemanha, com 04 estudos, e a Finlândia, com 03 estudos. China, Estados Unidos, França e Inglaterra, contemplam 02 estudos cada. Austrália, Bélgica, Botswana, Espanha, Holanda, Hong Kong, Reino Unido, Suécia, Taiwan e Turquia apresentam 01 estudo cada. Tem-se ainda 01 estudo realizado em 02 países, Alemanha e Luxemburgo. Um trabalho, particularmente, englobou dados de 13 países. Com relação à abrangência, 15 estudos foram realizados em domínio municipal, 10 estudos em abrangência nacional, 03 estudos foram pontuais, no caso em 01 universidade, 02 trabalhos em 02 ou mais países, 01 estudo de alcance regional e 01 intercontinental.

Todos os estudos tratam exclusivamente da profissão docente. No que tange à categoria de ensino onde os educadores lecionam, 07 estudos foram realizados com professores do ensino primário e secundário, 06 estudos com docentes do ensino secundário, 05 estudos com professores universitários, 04 estudos não especificam o grau do ensino, 03 estudos com docentes do ensino secundário ou escolas de gramática, 04 estudos com professores do ensino primário, 01 estudo com docentes de pré-escola, e 01 estudo com educadores de pré-escola e ensino.

O tamanho das populações pesquisadas apresentou grande amplitude, com variação de 10 a 10.000 docentes. Quanto ao gênero, 26 estudos pesquisaram professores e professoras, 04 estudos versam apenas de educadoras e 02 estudos não explicitam o gênero dos professores.

Quanto à abordagem metodológica, 01 estudo analítico, 02 qualitativos e 01 quali-quantitativo. Com relação ao desenho, 01 diário, 17 transversais e 02 longitudinais. No que tange ao objetivo, 01 exploratório e de desenvolvimento, 01 prospectivo, 01 interpretativo e 03 exploratórios. Sobre a técnica utilizada, 01 quase-experimento, 01 modelagem, 01 original, 01 estudo de múltiplos casos e 01 modelagem multinível.

Para a abordagem, convite e coleta dos dados foram adotadas diversas estratégias, tais quais o contato por telefone, e-mail, correio e pesso- 
Tabela 2. Características metodológicas dos estudos incluídos na revisão de literatura.

\begin{tabular}{|c|c|c|}
\hline Características & $\mathbf{n}$ & $\%$ \\
\hline \multicolumn{3}{|l|}{ País de origem do estudo } \\
\hline Brasil & 5 & 15,6 \\
\hline Alemanha & 4 & 12,5 \\
\hline Finlândia & 3 & 9,4 \\
\hline China & 2 & 6,3 \\
\hline Estados Unidos & 2 & 6,3 \\
\hline França & 2 & 6,3 \\
\hline Inglaterra & 2 & 6,3 \\
\hline Internacional & 2 & 6,3 \\
\hline Demais países com 1 estudo cada & 10 & 31,3 \\
\hline \multicolumn{3}{|l|}{ Abrangência } \\
\hline Institucional & 3 & 9,4 \\
\hline Municipal & 15 & 46,9 \\
\hline Regional & 1 & 3,1 \\
\hline Nacional & 10 & 31,3 \\
\hline Internacional & 3 & 9,4 \\
\hline \multicolumn{3}{|l|}{ Tipo de estudo } \\
\hline Longitudinal & 2 & 6,2 \\
\hline Transversal & 17 & 53,1 \\
\hline Não especifica & 13 & 40,6 \\
\hline \multicolumn{3}{|l|}{ Tamanho da amostra } \\
\hline$\leq 100$ & 5 & 15,6 \\
\hline $101-500$ & 11 & 34,4 \\
\hline $501-1000$ & 8 & 25 \\
\hline$\geq 1001$ & 5 & 15,6 \\
\hline Não especifica & 3 & 9,4 \\
\hline \multicolumn{3}{|l|}{ Coleta do dados } \\
\hline Questionário & 24 & 75,0 \\
\hline Entrevista & 2 & 6,3 \\
\hline Gravação da voz & 1 & 3,1 \\
\hline $\begin{array}{l}\text { Demais estudos com métodos } \\
\text { combinados }\end{array}$ & 5 & 15,6 \\
\hline \multicolumn{3}{|l|}{ Sexo dos participantes } \\
\hline Ambos & 26 & 81,3 \\
\hline Feminino & 4 & 12,5 \\
\hline Não especifica & 2 & 6,3 \\
\hline \multicolumn{3}{|l|}{ Nível de ensino } \\
\hline Professoras de pré-escola & 1 & 3,1 \\
\hline $\begin{array}{l}\text { Professores da pré-escola e ensino } \\
\text { primário }\end{array}$ & 1 & 3,1 \\
\hline $\begin{array}{l}\text { Professores da pré-escola, ensino } \\
\text { primário e secundário }\end{array}$ & 1 & 3,1 \\
\hline Professores do ensino primário & 4 & 12,5 \\
\hline $\begin{array}{l}\text { Professores do ensino primário e } \\
\text { secundário }\end{array}$ & 7 & 21,9 \\
\hline Professores do ensino secundário & 6 & 18,8 \\
\hline $\begin{array}{l}\text { Professores de escolas } \\
\text { secundárias ou de gramática }\end{array}$ & 3 & 9,4 \\
\hline Professores universitários & 5 & 15,6 \\
\hline Não especifica & 4 & 12,5 \\
\hline Total & 32 & 100,0 \\
\hline
\end{tabular}

Fonte: do Autor. almente. Os instrumentos de aferição foram, na maioria, questionários, combinados ou não, com entrevistas, gravações e exames clínicos.

Conforme descrito na Tabela 3, entre os temas principais dos artigos que compõem o portfólio, destaca-se a saúde ocupacional, abordada em 25 artigos. Ramifica-se em saúde mental, investigada em 20 oportunidades, tendo como assuntos pesquisados a Síndrome de Burnout e o estresse, em 06 estudos cada. A exaustão, a depressão e os distúrbios psíquicos também são tratados nas investigações. A saúde física aparece em outros 05 artigos, onde a voz é apontada em 04 trabalhos e a dor lombar é estudada uma vez.

Tabela 3. Assuntos e instrumentos de aferição descritos nos trabalhos incluídos na revisão de literatura.

\begin{tabular}{|c|c|c|}
\hline Características & $\mathbf{n}$ & $\%$ \\
\hline \multicolumn{3}{|l|}{ Saúde ocupacional } \\
\hline Saúde mental & 20 & 62,5 \\
\hline Saúde Física & 6 & 15,6 \\
\hline \multicolumn{3}{|l|}{ Ambiente de trabalho } \\
\hline Bem-estar & 4 & 12,5 \\
\hline Condições de trabalho & 3 & 9,4 \\
\hline Carga de trabalho & 2 & 6,3 \\
\hline Satisfação no trabalho & 2 & 6,3 \\
\hline \multicolumn{3}{|l|}{ Instrumentos } \\
\hline Job Content Questionnaire (JCQ) & 5 & 10,4 \\
\hline $\begin{array}{l}\text { General Health Questionnaire-12 } \\
\text { (GHQ-12) }\end{array}$ & 4 & 8,3 \\
\hline Maslach Burnout Inventory (MBI) & 4 & 8,3 \\
\hline CISS-S-2 & 3 & 6,3 \\
\hline $\begin{array}{l}\text { Effort-Reward Imbalance } \\
\text { Questionnaire (ERI) }\end{array}$ & 3 & 6,3 \\
\hline $\begin{array}{l}\text { Leiden Quality of Work } \\
\text { Questionnaire for teachers (LAKS- } \\
\text { DOC) }\end{array}$ & 3 & 6,3 \\
\hline $\begin{array}{l}\text { Maslach Burnout Inventory for } \\
\text { Educators (MBI-ES) }\end{array}$ & 3 & 6,3 \\
\hline $\begin{array}{l}\text { Somatization Scale of the Symptom } \\
\text { Checklist (SCL-90) }\end{array}$ & 3 & 6,3 \\
\hline C.A.G.E. & 2 & 4,2 \\
\hline $\begin{array}{l}\text { Occupational Stress Inventory } \\
\text { Revised Edition (OSI-R) }\end{array}$ & 2 & 4,2 \\
\hline $\begin{array}{l}\text { Self Reporting Questionnaire - } 20 \\
\text { (SRQ-20) }\end{array}$ & 2 & 4,2 \\
\hline $\begin{array}{l}\text { Structured Interview of Content } \\
\text { and Organisation of Work. JDC }\end{array}$ & 2 & 4,2 \\
\hline Work Ability Index (WAI) & 2 & 4,2 \\
\hline $\begin{array}{l}\text { Demais instrumentos com } 1 \\
\text { incidência cada }\end{array}$ & 35 & 72,9 \\
\hline
\end{tabular}


O ambiente de trabalho é assunto principal em 15 documentos, com destaque para o tópico bem-estar no trabalho, estudado em 04 oportunidades, e as condições de trabalho, investigadas em 03 estudos. As matérias carga de trabalho e satisfação aparecem em 02 trabalhos cada. Com 01 ocorrência consta a qualidade de vida, a capacidade para o trabalho, padrões de tempo de trabalho, horas extras e características do trabalho.

Além da coleta de dados pessoais, sociais e demográficos, que se amoldaram de acordo com o interesse das pesquisas, foram aplicados 47 questionários distintos, que mensuraram questões relacionadas ao trabalho, bem-estar e saúde. Destaque para o Job Content Questionnaire (JCQ), utilizado em 05 trabalhos, além do General Health Questionnaire-12 (GHQ-12) e do Maslach Burnout Inventory (MBI), utilizados em 04 oportunidades cada.

Os dados gerados pelas investigações foram analisados, na sua maioria (30 ocorrências), estatisticamente. Em 06 casos foi aplicada outra ferramenta de aferição, como análise qualitativa e quantitativa de diários, cargas alostáticas, análises bioquímicas, gravação da voz e ruídos, e utilização do software QSR NUD*IST para as unidades de texto. Um único estudo explorou as informações de maneira qualitativa e, para isso, fez uso do método de observação e análise de documentos, em três fases interpretativas: (1) uma análise de dados para cada entrevistado, (2) uma análise sistemática por escola e (3) uma análise comparativa de quatro escolas.

Relatos de limitações da pesquisa foram apontados em 24 trabalhos. Algumas restrições indicadas se referem à amostra, que por ser específica e homogênea, no caso de estudos realizados em apenas um estabelecimento, impedem a generalização dos achados; e em outros casos, por ser pequena, não se obteve representatividade. Outra limitação expressiva foi destacada em estudos transversais, que por incluírem apenas os sujeitos ativos, deixam de considerar os ausentes, até mesmo por motivo de doença, além de apresentar limitação para inferir uma relação de causalidade entre as variáveis consideradas.

Recomendações para pesquisas futuras foram sugeridas por 19 trabalhos, entre as quais, repetir o estudo com amostras distintas e ampliadas, examinar as relações abordadas em outros ambientes de ensino, incluir outros instrumentos de aferição, realizar pesquisas longitudinais sobre o mesmo tema, ampliar a abrangência do estudo e examinar os dados de maneira qualitativa.

\section{Discussão}

Embora os estudos selecionados apresentem diferentes delineamentos, tamanho de amostra e localidade, observou-se homogeneidade na preocupação com a oferta de melhores condições de trabalho para os docentes, de modo a auxiliar na manutenção do bem-estar, saúde e qualidade de vida desses profissionais. Para melhor compreensão e para atender ao interesse da pesquisa, a discussão dos resultados está subdivida em dois tópicos, são eles: Ambiente, organização e condições de trabalho, e Saúde do professor.

\section{Ambiente, organização e condições de trabalho}

No ambiente de trabalho, os desgastes são impostos ao ser humano basicamente pela organização e pelas exigências laborais ${ }^{18}$. Entre os principais fatores de influência depreciativa na vida laboral dos educadores, pode-se citar a carga horária elevada, como no caso Bentley e Kyvi ${ }^{4}$, em que professores universitários de 13 países, apresentam a média de 48,4 horas trabalhadas por semana, e cerca $20 \%$ dos docentes relataram trabalhar $60 \mathrm{~h}$ ou mais entre segunda e sexta-feira.

As horas extras e os serviços extraordinários também são fenômenos comuns e potencialmente capazes de atuar sobre a fadiga, o risco de lesões e a motivação dos professores. Ao reconhecer que estas são realidades do cotidiano do educador, o trabalho de Beckers et al. ${ }^{35}$ identificou que as escalas adicionais, se administradas de forma moderada, deixam de ser um problema entre os trabalhadores. Acordos flexíveis que forneçam controle do tempo de trabalho, além da alocação de horas-extras no início da semana, apresentam-se como medidas positivas na avaliação dos autores. Complementarmente, Marqueze et al. ${ }^{13}$ afirmam que a reestruturação administrativa sugestiona proficuamente a capacidade para o trabalho em professores.

Ainda de acordo com Marqueze et al. ${ }^{13}$, outra variável que apresenta correlação positiva com a aptidão laboral é a satisfação. Questão bastante investigada entre os docentes, o contentamento pode variar de acordo com o ambiente, a organização e as condições do trabalho a que os profissionais são expostos. O progresso acadêmico profissional, assim como o tempo de serviço, por exemplo, são aspectos associados com os níveis de satisfação em professores universitários tur$\cos ^{8}$. Para docentes de uma universidade privada 
americana, os recursos acadêmicos, a liderança, a influência e os suportes relacionais impactam no agrado laboral ${ }^{9}$.

Em estudos semelhantes, com amostras participantes de um projeto denominado EUROTE$\mathrm{ACH}$, que busca resultados sobre a qualidade de trabalho, bem-estar e saúde em professores, no primeiro deles de Griva e Joekes ${ }^{19}$, com docentes ingleses, foram relatados níveis mais baixos de bem-estar psicológico e físico do que a média europeia. Os investigados apresentam sintomas mais elevados de burnout e queixas somáticas, bem como menor satisfação no trabalho. Em consonância com isso, eles também avaliam os aspectos de seu trabalho mais negativamente do que os seus colegas europeus o fazem, percebem mais riscos ambientais e demandas relacionados ao trabalho, têm menos senso de controle e entendem enfrentar níveis mais elevados de esforço físico, além de horários de trabalho mais longos. $\mathrm{Na}$ contramão, o estudo de Rasku e Kinnunen ${ }^{39}$ expõe que as condições de trabalho e o bem-estar dos professores finlandeses de ensino secundário se destacam positivamente frente aos seus colegas europeus. Muito disso se deve aos escores mais altos de controle e satisfação no trabalho, além de pontuações mais baixas acerca das exigências laborais, despersonalização e queixas somáticas. Apesar dos pesquisados, na Finlândia, relatarem trabalhar mais horas por semana do que a média europeia, estes resultados positivos podem ser um indicativo das práticas de proteção ao bem - estar do trabalhador, realizadas no país ao longo da história.

Apesar dos resultados favoráveis encontrados no cenário finlandês, a realidade das condições de trabalho a que são submetidos os profissionais de ensino é geralmente precária. Diversos estudos tratam a profissão de educador como uma das mais difíceis ${ }^{46}$, desafiadoras ${ }^{44}$, estressoras ${ }^{40} \mathrm{e}$ potencialmente expostas a fatores de risco à saúde no mundo 5 .

Os estudos de Yang et al ${ }^{40}$ reforçam o quão árduo é o cenário de uma amostra de docentes do ensino primário e secundário na China, que apresentaram o índice de qualidade de vida menor do que a população chinesa em geral, além do que, em professoras, esse número é menor em relação aos professores, e decrescente com o avanço da idade.

Ainda com relação ao ambiente e às condições de trabalho, a categoria é dada como uma das mais expostas a situações conflituosas ${ }^{2}$. De acordo com Bauer et $\mathrm{al} .{ }^{46}$ um número considerável de docentes alemães, que participaram de sua pesquisa, reportou já terem passado pela experiência de vandalismo, sofrido ameaças ou violência por parte de alunos ou pais. Essa situação, considerada por Unterbrink et al. ${ }^{31}$ como social e sem perspectivas de transformação a curto prazo no país, é passível de resolução a partir da promoção de suporte social como fator preventivo para a manutenção do bem-estar docente, além de se mostrar uma oportunidade para o desenvolvimento de novas competências para lidar com essas dificuldades. $\mathrm{O}$ ambiente escolar e o apoio têm sua importância salientada por Kovess -Masféty et al. ${ }^{43}$, no controle da saúde mental em professores de escolas públicas francesas.

\section{Saúde do professor}

O tema saúde ocupacional relacionado aos docentes pode ser abordado através de duas frentes, a física e a mental. Os artigos selecionados para compor o portfólio exploram principalmente o bem-estar psíquico e cognitivo desses trabalhadores. Porém, a saúde física também é destaque primário em alguns trabalhos e secundário em outros, inclusive como vetores de distúrbios mentais.

A título de exemplo, esforços físicos realizados no trabalho foram frequentemente apontados pelos professores brasileiros de pré-escola, ensino primário e secundário ${ }^{18}$, que destacaram as posturas incômodas e inadequadas do corpo, no caso, permanecer em pé ou correção de trabalhos escolares por tempo demasiado, como fatores de desgaste. No trabalho de Erick e Smith $^{16}$, que pesquisou a dor lombar em docentes do ensino primário e secundário de Botswana, os investigados relataram que seu trabalho exige alto esforço físico, atividade física rápida, posturas incômodas de corpo e braço. Essas demandas, aliadas às altas exigências psicológicas, apresentaram associação com a dor lombar. Já o exercício físico regular manteve-se conexo com a diminuição nas chances de manifestação desse tipo de algia; a maioria dos professores com dor lombar $(67,1 \%)$ relataram incapacidade mínima; entre os aspectos individuais, gênero, idade, índice de massa corporal, nível de escolaridade e de baixa lesão nas costas anterior foram significativamente associados com a dor lombar.

Os distúrbios da voz também são partilhados pela profissão docente e bastante enfocados nos estudos que envolvem esta categoria. Educadores com alteração vocal, do ensino primário e secundário de Taiwan ${ }^{40}$, mostraram-se predispostos a tomar medicamentos, sofrer de infecção das vias 
respiratórias superiores, o que resulta, em de forma complementar, em estresse e ansiedade. Professoras brasileiras do ensino primário ${ }^{34}$ citaram ter tido licença do trabalho devido a enfermidades relacionadas à voz; houve relatos frequentes de condições, tais como sinusite, rinite, amigdalite, faringite; ou queixas de barulho nas salas de aula e ruído gerado na escola o que, consequentemente, acaba por acarretar em disfonia, devido à necessidade de aumentar a voz. No estudo com professoras suecas de pré-escola ${ }^{27}$, foi utilizado o recurso de gravação do som, expondo que o nível médio do ruído de fundo em sala de aula estava $20 \mathrm{~dB}$ acima do que é recomendado, o que revelou a necessidade de redução dos níveis de ruído do ambiente, além do aumento do número de pausas ou intervalos. A investigação de Rantala et al. ${ }^{45}$ salienta que disciplinas como fisiologia do exercício e ergonomia ocupacional são as que mais extensivamente têm estudado a fadiga vocal.

As questões mentais ocupam o lugar de maior destaque na agenda de pesquisa quando o tema é condições de trabalho e saúde docente. O trabalho de Gasparini et al. ${ }^{3}$ realizado com professores primários brasileiros apontou forte associação entre a prevalência de transtornos mentais e variáveis relacionadas à experiência de violência na escola, à percepção negativa sobre o trabalho e às condições do ambiente físico na escola, cita-se ruído, ventilação e situação das paredes das salas de aula.

Os distúrbios psíquicos menores são investigados, de modo genérico, no estudo de Reis et al. ${ }^{7}$, e específico, com foco nos sintomas depressivos, no trabalho de Kidger et al. ${ }^{44}$. Entretanto, estresse e burnout - que conforme Bellingrath et al. ${ }^{37}$ podem inclusive aparecer relacionados - são mesmo os assuntos em evidência entre os pesquisadores da área e essa condição se confirma através dos artigos selecionados no portfólio.

A pesada carga de trabalho vivenciada por professores de Hong Kong está diretamente relacionada com a sua rotina diária de ensino, onde fatores ligados aos estudantes, às pressões e expectativas, atribuições adicionais e reconhecimento são consideradas fontes de estresse ${ }^{20}$. Além das variáveis ligadas ao ambiente, a personalidade e as diferenças individuais são fundamentais para entender por que algumas pessoas sofrem de estresse relacionado com o trabalho. Essa contribuição pessoal é investigada em docentes de ensino primário e secundário do Reino Unido, onde a primeira classe percebeu maiores níveis de estresse do que os colegas da segunda categoria $^{30}$.
É possível, no entanto, que a intervenção gerencial modifique e controle muitos dos fatores de influência no estresse ocupacional, através de programas de prevenção. Os resultados da intervenção realizada na pesquisa de Wu et al. ${ }^{38}$ mostram que o ajuste do ambiente ao indivíduo diminuiu os índices de atuação da sobrecarga, limites impostos à função, responsabilidade e ambiente físico sobre o estresse, o que aumentou, por consequência o índice de capacidade para o trabalho dos professores chineses pesquisados.

A Síndrome de Burnout, por sua vez, é significativamente relacionada com as condições de trabalho a que os docentes são submetidos, principalmente em ambientes precários de ensino, que geram aumentos significativos nos níveis de esgotamento $^{21}$. O nível de ensino, uma vizinhança socioeconomicamente desprivilegiada e o número de alunos são determinantes potenciais de burnout em professores franceses ${ }^{15}$. As interações sociais são reportadas como as mais desafiadoras e problemáticas questões em termos de exaustão relacionada ao trabalho, em educadores finlandeses. Outra relação estreita verificada pela pesquisa de Pas et al. ${ }^{42}$ é entre os baixos índices de eficácia do docente e o aumento dos níveis de burnout com o passar do tempo.

$\mathrm{Na}$ pesquisa de Unterbrink et al. ${ }^{36}$ um alto percentual de professores apresentam sintomas de ambos burnout e alto esforço com baixa recompensa. Demonstra-se que essa situação invoca medidas preventivas e a necessidade de mudanças em aspectos da estrutura do ambiente, como tamanho das salas de aula e número de alunos, além de suporte social para o desenvolvimento de habilidades interpessoais para lidar com as dificuldades de relacionamento com pais e estudantes.

\section{Considerações finais}

Demonstra-se, dessa maneira, que a classe docente, em sua totalidade, possui a convergência da transmissão do conhecimento como ofício. No entanto, são as particularidades do nível e ambiente de ensino que singularizam as peculiaridades da natureza desta profissão. Os estudos detalhados e discutidos nesta revisão evidenciaram as preocupações existentes diante de fatores nocivos à saúde e ao trabalho dos professores. Constaram pesquisas com educadores da pré-escola à universidade, de diversos países, que abordaram condições de trabalho e sintomas somáticos e psicossomáticos. 
Este contexto de investigação é repleto de demandas das quais, em termos de ambiente profissional, destacaram-se o bem-estar e a carga horária. Com relação à saúde do professor, foi possível identificar um predomínio de análises que ponderam o aspecto mental do indivíduo, com ênfase para o estresse e a Síndrome do Bur- nout. Os distúrbios da voz também foram evidenciados em diversos trabalhos. Tão imprescindível quanto à identificação das causas e efeitos destes desgastes é a readequação das atividades e do cotidiano escolar ao docente, conceito este pertinente às concepções da ergonomia.

\section{Colaboradores}

Ideia e formulação do estudo: JG Luz, SLR Pessa. Orientação da pesquisa e apresentação dos métodos: SLR Pessa, FJA Schenatto. Coleta e análise de dados: JG Luz, RP Luz. Redação do artigo: JG Luz. Revisão crítica e revisão do artigo: JG Luz, FJA Schenatto, RP Luz. 


\section{Referências}

1. Klusmann U, Kunter M, Trautwein U, Ludtke O, Baumert J. Engagement and Emotional exhaustion in teachers: Does the school context make a difference? Appl Psycho 2008; 57(1):127-151.

2. Pyhältö K, Pietarinen J, Salmela-Aro K. Teacher-working-environment fit as a framework for burnout experienced by Finnish teachers. Teach Teach Educ 2011; 27(7):1101-1110.

3. Gasparini M, Barreto SM, Assunção AA. Prevalência de transtornos mentais comuns em professores da rede municipal de Belo Horizonte, Minas Gerais, Brasil. Cad Saude Publica 2006; 22(12):2679-2691.

4. Bentley PJ, Kyvik S. Academic work from a comparative perspective: a survey of faculty working time across 13 countries. Higher Education 2012; 63(4):529-547.

5. Pascual E, Perez-Jover V, Mirambell E, Ivañez G, Terol MC. Job conditions, coping and wellness/health outcomes in spanish secondary school teachers. Psychology and Health 2003; 18(4):511-521.

6. Ballet K, Kelchtermans G. Struggling with workload: Primary teachers' experience of intensification. Teach Teach Educ 2009; 25(8):1150-1157.

7. Reis EJFB, Carvalho FM, Araújo TM, Porto LA, Neto AMS. Trabalho e distúrbios psíquicos em professores da rede municipal de Vitória da Conquista, Bahia, Brasil. Cad Saude Publica 2005; 21(5):1480-1490.

8. Filiz Z. An analysis of the levels of job satisfaction and life satisfaction of the academic staff. Soc Indic Res 2014; 116(3):793-808.

9. Bilimoria D, Perry SR, Liang X, Stoller EP, Higgins P, Taylor C. How do female and male faculty members construct job satisfaction? The roles of perceived institutional leadership and mentoring and their mediating processes. J Technol Transf 2006; 31(3):355-365.

10. Judge TA, Heller D, Mount MK. Five-factor model of personality and job satisfaction: A meta-analysis. J Appl Psychol 2002; 87(3):530-541.

11. Kristof-Brown AL, Zimmerman RD, Johnson EC. Consequences of individuals' fit at work: a meta-analysis of person-job, person-organization, persongroup, and person-supervisor fit. Pers Psychol 2005; 58(2):281-342.

12. Linden DVD, Taris TW, Beckers DGJ, Kindt KB. Reinforcement sensitivity theory and occupational health: BAS and BIS on the job. Personality and Individual Differences 2007; 42(2):1127-1138.

13. Marqueze EC, Voltz GP, Borges FN, Moreno CR. A 2-year follow-up study of work ability among college educators. Appl Ergon 2008; 39(5):640-645.

14. Meyer JP, Hecht TD, Gill H, Toplonytsky L. Personorganization (culture) fit and employee commitment under conditions of organizational change: A longitudinal study. J Vocat Behav 2010; 73(3):458-473.

15. Vercambre MN, Brosselin P, Gilbert F, Nerrière E, Kovess-Masféty V. Individual and contextual covariates of burnout: A cross-sectional nationwide study of french teachers. BMC Public Health 2009; 9:333.

16. Erick PN, Smith DR. Low back pain among school teachers in Botswana, prevalence and risk factors. BMC Musculoskelet Disord 2014; 15:359.
17. Tuomi K, Huuhtanen P, Nykyri E, Ilmarinen J. Promotion of work ability, the quality of work and retirement. Occup Med (Lond) 2001; 51(5):318-324.

18. Delcor NS, Araújo TM, Reis EJFB, Porto LA, Carvalho FM, Silva MO, Barbalho L, Andrade JM. Condições de trabalho e saúde dos professores da rede particular de ensino de Vitória da Conquista, Bahia, Brasil. Cad Saude Publica 2004; 20(1):187-196.

19. Griva K, Joekes K. UK teachers under stress: Can we predict wellness on the basis of characteristics of the teaching job? Psychology and Health 2003; 18(4):457471.

20. Jin P, Yeung AS, Tang T, Low R. Identifying teachers at risk in Hong Kong: psychosomatic symptoms and sources of stress. J Psychosom Res 2008; 65(4):357-362.

21. Goddard, R, O'brien P, Goddard M. Work environment predictors of beginning teacher burnout. $\mathrm{Br}$ Educ Res J 2006; 32(6):857-874.

22. Stoeber J, Rennert D. Perfectionism in school teachers: Relations with stress appraisals, coping styles, and burnout. Anxiety Stress Coping 2008; 21(1):37-53.

23. Swider BW, Zimmerman RD. Born to burnout: A meta-analytic path model of personality, job burnout, and work outcomes. J Vocat Behav 2010; 76(3):487506.

24. Simberg S, Sala E, Rönnemaa AM. A comparison of the prevalence of vocal symptoms among teacher students and other university students. J Voice 2004; 18(3):363-368

25. Simberg S, Sala E, Vehmas K, Laine A. Changes in the prevalence of vocal symptoms among teachers during a twelve-year period. J Voice 2005; 19(1):95-102.

26. Sliwinska-Kowalska M, Niebudek-Bogusz E, Fiszer M, Los-Spychalska T, Kotylo P, Sznurowska-Przygocka $B$, Modrzewska $M$. The prevalence and risk factors for occupational voice disorders in teacher. Folia Phoniatr Logop 2006; 58(2):85-101.

27. Södersten M, Granqvist S, Hammarberg B, Szabo A. Vocal behavior and vocal loading factors for preschool teachers at work studied with binaural DAT recordings. J Voice 2002; 16(3):356-371.

28. Cheng HYK, Wong MT, Yu YC, Ju YY. Work-related musculoskeletal disorders and ergonomic risk factors in special education teachers and teacher's aides. BMC Public Health 2016; 16:137.

29. Kathy Cheng HY, Cheng CY, Ju YY. Work-related musculoskeletal disorders and ergonomic risk factors in early intervention educators. Appl Ergon 2013; 44(1):134-141.

30. Jepson E, Forrest S. Individual contributory factors in teacher stress: The role of achievement striving and occupational commitment. Br J Educ Psychol 2006; 76(Pt 1):183-197.

31. Unterbrink T, Zimmermann L, Pfeifer R, Wirsching M, Brähler E, Bauer J. Parameters influencing health variables in a sample of 949 german teachers. Int Arch Occup Environ Health 2008; 82(1):117-123.

32. Liberati A, Altman DG, Tetzlaff J, Mulrow C, Gotzche PC, Ioannidis JPA, Clarke M, Devereaux PJ, Kleijnen J, Moher D. The PRISMA statement for reporting systematic reviews and meta-analyses of studies that evaluate health care interventions: Explanation and elaboration. PLoS Med 2009; 6(7):e1000100. 
33. Moher D, Liberati A, Tetzlaff J, Altman DG. Preferred reporting items for systematic reviews and meta-analyses: The PRISMA statement. PLoS Med 2009; 6(7):264-269.

34. Medeiros AM, Barreto SM, Assunção AA. Voice disorders (dysphonia) in public school female teachers working in Belo Horizonte: Prevalence and associated factors. J Voice 2008; 22(6):676-687.

35. Beckers DG, van Hooff ML, van der Linden D, Kompier MA, Taris TW, Geurts SA. A diary study to open up the black box of overtime work among university faculty members. Scand J Work Environ Health 2008; 34(3):213-223.

36. Unterbrink T1, Hack A, Pfeifer R, Buhl-Griesshaber V, Müller U, Wesche H, Frommhold M, Scheuch $\mathrm{K}$, Seibt R, Wirsching M, Bauer J. Burnout and effortreward-imbalance in a sample of 949 german teachers. Int Arch Occup Environ Health 2007; 80(5):433-441.

37. Bellingrath S1, Weigl T, Kudielka BM. Chronic work stress and exhaustion is associated with higher allostastic load in female school teachers. Stress 2009; 12(1):37-48.

38. Wu S, Li J, Wang M, Wang Z, Li H. Intervention on occupational stress among teachers in the middle schools in China. Stress Health 2006; 22(5):329-336.

39. Rasku A, Kinnunen U. Job conditions and wellness among finnish upper secondary school teachers. Psychology \& Health 2003; 18(4):441-456.

40. Yang X, Ge C, Hu B, Chi T, Wang L. Relationship between quality of life and occupational stress among teachers. Public Health 2009; 123(11):750-755.

41. Chen SH, Chiang SC, Chung YM, Hsiao LC, Hsiao TY. Risk factors and effects of voice problems for teachers. J Voice 2010; 24(2):183-190.

42. Pas ET1, Bradshaw CP, Hershfeldt PA. Teacher-and school-level predictors of teacher efficacy and burnout: Identifying potential areas for support. J Sch Psychol 2012; 50(1):129-145.
43. Kovess-Masféty V, Rios-Seidel C, Sevilla-Dedieu C. Teachers' mental health and teaching levels. Teach Teach Educ 2007; 23(7):1177-1192.

44. Kidger J, Brockman R, Tilling K, Campbell R, Ford T, Araya R, King M, Gunnell D. Teachers' wellbeing and depressive symptoms, and associated risk factors: A large cross sectional study in english secondary schools. J Affect Disord 2016; 192:76-82.

45. Rantala L, Vilkman E, Bloigu R. Voice changes during work: subjective complaints and objective measurements for female primary and secondary schoolteachers. J Voice 2002 16(3):344-355.

46. Bauer J, Unterbrink T, Hack A, Pfeifer R, BuhlGriesshaber V, Müller U, Wesche H, Frommhold M, Seibt R, Scheuch K, Wirsching M. Working conditions, adverse events and mental health problems in a sample of 949 german teachers. Int Arch Occup Environ Health 2007; 80(5):442-449.

Artigo apresentado em 19/10/2017

Aprovado em15/05/2018

Versão final apresentada em 17/05/2018 